\title{
Magnetic dimerization in the frustrated spin ladder $\mathrm{Li}_{2} \mathrm{Cu}_{2} \mathrm{O}\left(\mathrm{SO}_{4}\right)_{2}$
}

\author{
O. Vaccarelli, ${ }^{1}$ G. Rousse, ${ }^{2}$ A. Saúl, ${ }^{3}$ and G. Radtke ${ }^{1, *}$ \\ ${ }^{1}$ Institut de Minéralogie, de Physique des Matériaux, et de Cosmochimie (IMPMC), Sorbonne Universités-UPMC Univ Paris 06, UMR CNRS \\ 7590, Muséum National d'Histoire Naturelle, IRD UMR 206, 4 place Jussieu, F-75005 Paris, France \\ ${ }^{2}$ Collège de France, Chimie du Solide et de l'Energie, UMR 8260, 11 place Marcelin Berthelot, 75231 Paris Cedex 05, France \\ ${ }^{3}$ CINaM, UMR 7325 CNRS, Aix-Marseille University, Campus de Luminy, 13288 Marseille Cedex 9, France
}

(Received 3 April 2017; published 13 November 2017)

\begin{abstract}
The magnetic properties of $\mathrm{Li}_{2} \mathrm{Cu}_{2} \mathrm{O}\left(\mathrm{SO}_{4}\right)_{2}$ are investigated in the framework of density functional theory. In its high-temperature tetragonal structure, this compound appears as a rare material realization of a frustrated spin-1/2 two-leg ladder, where magnetic frustration arises from competing nearest and next-nearest interactions along the legs. Through a large magnetoelastic coupling, the triclinic distortion occurring around $125 \mathrm{~K}$ is shown to induce the formation of a staggered dimer structure, lifting most of the magnetic frustration.
\end{abstract}

DOI: 10.1103/PhysRevB.96.180406

During the last few decades, a considerable effort has been devoted to the experimental and theoretical investigation of frustrated quantum antiferromagnets [1]. Among the different models studied as potential candidates in which new states of matter could occur, the frustrated $S=1 / 2$ two-leg spin ladder has received considerable attention, as it combines low spin, low dimensionality, and magnetic frustration. The general Hamiltonian for this model can be written as

$$
\begin{aligned}
H= & J \sum_{\alpha=1,2} \sum_{i} \mathbf{S}_{\alpha, i} \cdot \mathbf{S}_{\alpha, i+1}+J_{\perp} \sum_{i} \mathbf{S}_{1, i} \cdot \mathbf{S}_{2, i} \\
& +J_{\times} \sum_{i}\left(\mathbf{S}_{1, i} \cdot \mathbf{S}_{2, i+1}+\mathbf{S}_{1, i+1} \cdot \mathbf{S}_{2, i}\right) \\
& +J_{2} \sum_{\alpha=1,2} \sum_{i} \mathbf{S}_{\alpha, i} \cdot \mathbf{S}_{\alpha, i+2},
\end{aligned}
$$

where the index $\alpha$ distinguishes left and right legs, $i$ labels rungs, and the $\mathbf{S}_{\alpha, i}$ are the $S=1 / 2$ operators at the rung $i$ on the $\alpha^{\text {th }}$ leg of the ladder. $J$ is the nearest-neighbor (NN) exchange coupling along the legs and $J_{\perp}$ the interleg coupling along the rungs. Frustration arises either through the nextnearest-neighbor (NNN) coupling along the legs $J_{2}$ or through the diagonal, interleg coupling $J_{\times}$.

In the absence of next-nearest-neighbor coupling $\left(J_{2}=0\right)$, the phase diagram for this model was originally shown to consist of two parts: a Haldane and a rung-singlet phase [2-4]. It was later suggested that this picture might be incomplete and that an additional, intermediate dimerized phase could also occur [5,6]. Vekua and Honecker [7] further showed that the addition of sufficiently strong next-nearest-neighbor couplings along the legs $\left(J_{2} \neq 0\right)$ stabilizes additional columnar dimer and staggered dimer phases. A rich phase diagram thus emerges from this quasi-one-dimensional (quasi-1D) lattice model depending critically on the relative signs and strengths of the various exchange couplings.

Unfortunately, only a very limited number of material systems can be considered as true realizations of frustrated $S=1 / 2$ two-leg spin ladders and thus, provide experimental

\footnotetext{
*guillaume.radtke@upmc.fr
}

evidence to be confronted by these theoretical predictions. Whereas $\mathrm{SrCu}_{2} \mathrm{O}_{3}$ [8] or $\mathrm{Sr}_{14} \mathrm{Cu}_{24} \mathrm{O}_{41}[9,10]$ are prototypical realizations of nonfrustrated ladders, $\mathrm{BiCu}_{2} \mathrm{PO}_{6}$ [11] might appear as one of the very rare examples of $S=1 / 2$ frustrated spin ladder where the frustration arises only from NNN interactions along the legs [12].

In this Rapid Communication, we show that the newly synthesized compound $\mathrm{Li}_{2} \mathrm{Cu}_{2} \mathrm{O}\left(\mathrm{SO}_{4}\right)_{2}$ [13] is an actual realization of $S=1 / 2$ frustrated two-leg spin ladder. Moreover, we demonstrate that the tetragonal to triclinic structural transition occurring around $125 \mathrm{~K}$ [14] leads to the emergence of a staggered $S=1 / 2$ dimer structure, lifting most of the magnetic frustration.

The crystal structure of $\mathrm{Li}_{2} \mathrm{Cu}_{2} \mathrm{O}\left(\mathrm{SO}_{4}\right)_{2}$ at room temperature is shown in Figs. 1(a) and 1(b). This compound crystallizes in a tetragonal structure, with space group $P 4_{2} / \mathrm{m}$ where $\mathrm{Cu}^{2+}$ ions occupy a slightly distorted square planar environment, as commonly observed for this strong JahnTeller ion. The resulting $\mathrm{CuO}_{4}$ squares are grouped by two, sharing an edge to form $\mathrm{Cu}_{2} \mathrm{O}_{6}$ platelets. These platelets are connected one to each other through an oxygen atom, after being rotated by $90^{\circ}$ under the effect of the $4_{2}$ helical axis leading to infinite $\mathrm{Cu}_{2} \mathrm{O}_{5}$ chains running along the $c$ axis of the crystal [see Fig. 1(b)]. Tetrahedral $\mathrm{SO}_{4}$ units further link every second platelet along the chains by sharing two oxygen ions with them. These chains are finally separated from each other by the $\mathrm{Li}^{+}$ions. From a magnetostructural perspective, dominant magnetic couplings should occur in this structure either through intra- $\left[J_{\perp}\right.$ in Fig. $\left.1(\mathrm{c})\right]$ or interplatelets $\left(J=J_{\times}\right) \mathrm{Cu}-\mathrm{O}-\mathrm{Cu}$ superexchange mechanisms or through longer ranged interactions via the nonmagnetic bridging $\mathrm{SO}_{4}$ units $\left(J_{2}\right)$, as such polyanionic groups are known to be efficient media for magnetic interactions [15]. As additional interchain interactions are expected to be weak due to the absence of well-defined covalent superexchange paths, this compound should exhibit a strong quasi-1D character. Note that the resulting geometry for such an isolated chain maps exactly on a $S=1 / 2$ frustrated two-leg ladder described by (1) in the special case where $J=J_{\times}$as illustrated in Fig. 1(d).

Figure 2 shows the paramagnetic band structure and density of states (DOS) of $\mathrm{Li}_{2} \mathrm{Cu}_{2} \mathrm{O}\left(\mathrm{SO}_{4}\right)_{2}$ calculated close to the Fermi level. These density functional theory (DFT) 


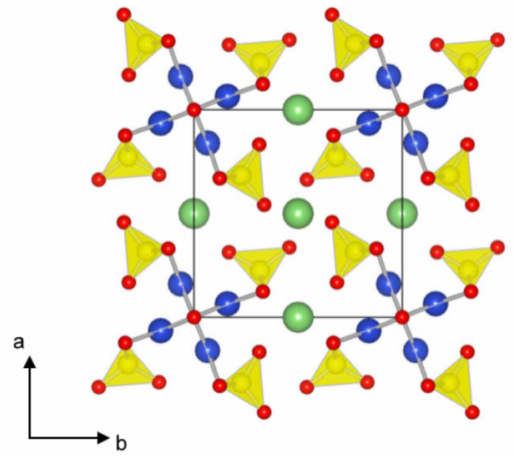

(b)

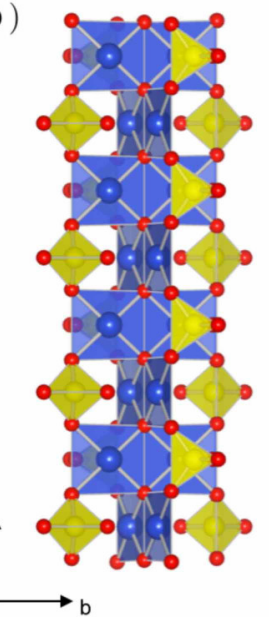

(c)

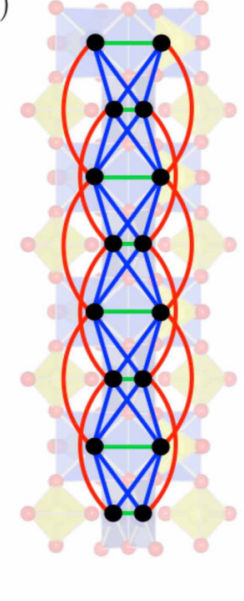

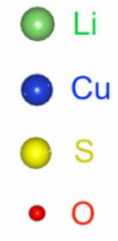

(d)

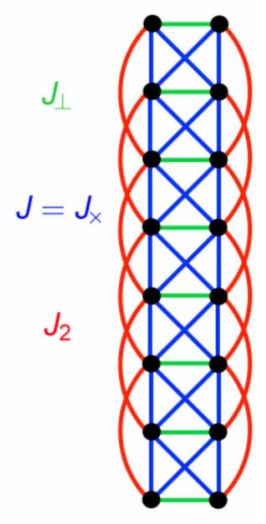

FIG. 1. (a) Tetragonal crystal structure of $\mathrm{Li}_{2} \mathrm{Cu}_{2} \mathrm{O}\left(\mathrm{SO}_{4}\right)_{2}$ at room temperature. $\mathrm{Cu}$ are in blue, $\mathrm{O}$ in red, $\mathrm{S}$ in yellow, and $\mathrm{Li}$ in green. (b) Detail of the atomic structure of the chains running along the $c$ axis. (c) Magnetic model deduced from the atomic structure, with the three dominant interactions along the chain: $J_{\perp}$ in green, $J=J_{\times}$in blue, and $J_{2}$ in red. (d) Topologically equivalent frustrated two-leg spin ladder.

calculations have been carried out using the pseudopotential plane-wave method as implemented in the QUANTUM ESPRESSO suite of codes [16]. Exchange and correlation has been accounted for in the generalized gradient approximation (GGA) parametrized by Perdew, Burke and Ernzerhof (PBE) [17]. Ultrasoft pseudopotentials [18] have been employed with a plane-wave and charge density cutoffs of 60 and $480 \mathrm{Ry}$, respectively. The four half-filled bands of dominant $\mathrm{Cu}-3 d_{x^{2}-y^{2}}$ character hybridized with the $2 p$ states of the neighboring oxygen ions are well separated from the continuum manifold. Strikingly, these bands are almost dispersionless except along $\Gamma-Z$, i.e., along the chain direction, confirming therefore the marked quasi-1D character of these electronic states, expected from structural considerations. Maximally localized Wannier function [19] (MLWF) interpolation of the band structure was performed using WANNIER90 [20] and is shown in Fig. 2. This interpolation allows the extraction of the effective hopping integrals between magnetic orbitals and reveals that three interactions largely dominate the dispersion: the intraplatelet hopping $t_{\perp}=-146 \mathrm{meV}$, the $\mathrm{NN}$ interplatelet hopping $t=$ $161 \mathrm{meV}$, and the NNN hopping along the legs $t_{2}=101 \mathrm{meV}$. Considering these three terms only, a tight-binding description of the band structure can easily be constructed leading to the

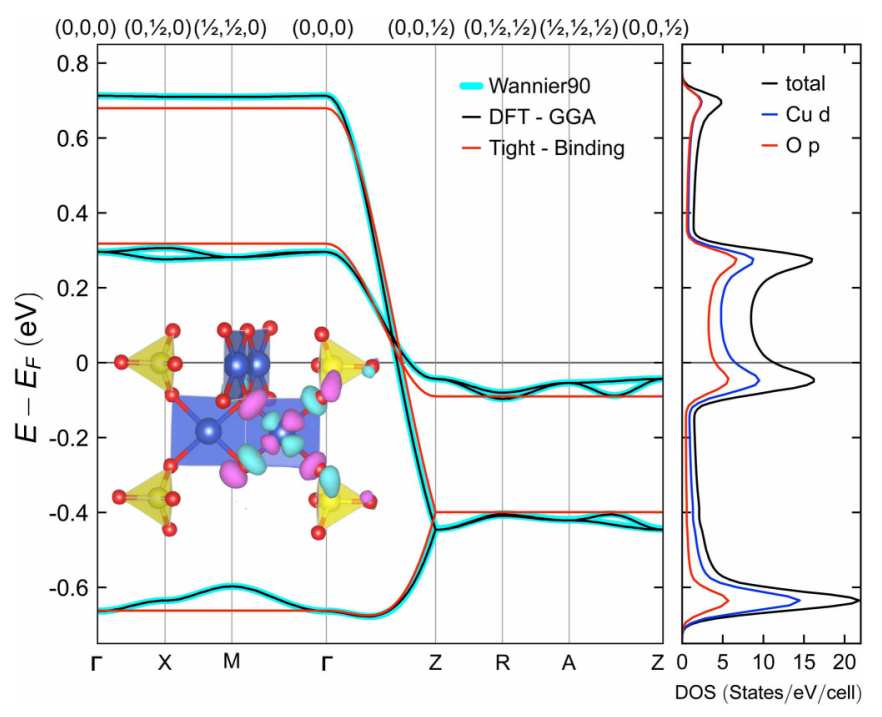

FIG. 2. Detail of the paramagnetic $\mathrm{Cu}-3 d_{x^{2}-y^{2}}$ band manifold around the Fermi level in $\mathrm{Li}_{2} \mathrm{Cu}_{2} \mathrm{O}\left(\mathrm{SO}_{4}\right)_{2}$ calculated using GGA-PBE and interpolated with MLWFs (left panel) and corresponding total and partial density of states (right panel). The inset shows one of the MLWFs centered on the $\mathrm{Cu}$ site; the large antibonding $\mathrm{O}-2 p$ tails are clearly visible on neighboring atoms.

analytical results

$$
\begin{aligned}
\epsilon_{1,2}(\mathbf{k}) & =\epsilon_{3 d}-t_{\perp}+2 t_{2} \cos \left(2 \pi k_{z}\right), \\
\epsilon_{3}(\mathbf{k}) & =\epsilon_{3 d}+t_{\perp}-4 t \cos \left(\pi k_{z}\right)+2 t_{2} \cos \left(2 \pi k_{z}\right), \\
\epsilon_{4}(\mathbf{k}) & =\epsilon_{3 d}+t_{\perp}+4 t \cos \left(\pi k_{z}\right)+2 t_{2} \cos \left(2 \pi k_{z}\right) .
\end{aligned}
$$

The corresponding bands are represented in Fig. 2 with red lines and clearly illustrate the excellent description of the electronic structure provided by this simplified 1D model. A mapping of the paramagnetic band structure onto a single-band Hubbard model at half-filling, eventually reducing to an antiferromagnetic (AFM) Heisenberg model in the strongly correlated limit, provides a direct link between these hopping parameters and the AFM component of the magnetic couplings, through the expression $J^{\mathrm{AFM}}=4 t^{2} / U_{\text {eff }}$. One could therefore expect the three dominant couplings $J_{\perp}, J$, and $J_{2}$ to be essentially AFM and of the same order of magnitude. However, this simple analysis overlooks the presence of potentially large ferromagnetic (FM) contributions [21-23] which, depending on the detailed atomic arrangement supporting the superexchange mechanisms, could partially balance or even dominate their AFM counterparts. $J$ is primarily associated with a $\mathrm{Cu}-\mathrm{O}-\mathrm{Cu}$ bond forming an angle of $116^{\circ}$ and is likely to be dominated by its AFM component. $J_{2}$ corresponds to a long-range interaction mediated by a bridging $\mathrm{SO}_{4}$ group, a geometry which usually favors antiferromagnetism too and might give rise to strong couplings $[15,24]$. The situation is, however, very different for $J_{\perp}$ where the $\mathrm{Cu}-\mathrm{O}-\mathrm{Cu}$ bond forms an angle of $97^{\circ}$, close to the FM-AFM crossover [25].

In order to investigate this point, the magnetic couplings were estimated within the broken symmetry formalism, i.e., by mapping total energies corresponding to various collinear spin arrangements within a supercell $[15,26]$ onto 
the Heisenberg Hamiltonian (1) with $J=J_{\times}$by symmetry. Total energies were calculated in $\mathrm{GGA}+U$ to improve the treatment of strongly correlated $\mathrm{Cu}-3 d$ electrons. A value of $U_{\text {scf }}=10.85 \mathrm{eV}$ for the effective self-consistent Hubbard term was determined for a $1 \times 1 \times 2$ supercell following the approach described in Refs. [27,28]. The mapping has been carried out using the total energies of 42 spin configurations calculated in a $1 \times 1 \times 2$ supercell containing four formula units and based on the experimental structure determined at $300 \mathrm{~K}$ (see Supplemental Material [29]). The results give $J=J_{\times} \approx J_{2}=127 \mathrm{~K}$ confirming the expected AFM nature of these couplings. Note here that if the first equality is dictated by symmetry, the second is only numerical. Finally, $J_{\perp}=-100 \mathrm{~K}$; the intraplatelet coupling is ferromagnetic. Additional calculations performed using a $2 \times 1 \times 1$ supercell confirm the absence of sizable interladder couplings.

The picture emerging from the calculated effective exchange interactions is therefore that of a quasi-1D and highly frustrated spin ladder system, where the frustration arises from competing NN and NNN interactions along the legs.

This image is confirmed by the numerical calculation of the ground-state expectation values of the spin correlations $\left\langle\mathbf{S}_{i} \cdot \mathbf{S}_{j}\right\rangle$ using Lanczos diagonalization on a finite lattice of 24 spins and imposing periodic boundary conditions. Finite-size effects were found to be small, particularly for the dimerized phase. In agreement with the FM nature of $J_{\perp}$, the intraplatelet value of 0.241 is very close to the value expected for a triplet, i.e., 0.250. Values for the $\mathrm{NN}$ and $\mathrm{NNN}$ interactions along the legs of -0.269 and -0.046 reveal frustrated quasi-1D antiferromagnetic correlations. Setting $J_{2}=0$ is enough to entirely lift the magnetic frustration. The intraplatelet, $\mathrm{NN}$, and NNN interactions then reach $0.250,-0.351$, and 0.194 , respectively, consistent with a Haldane ground state [2].

As recently reported, this compound undergoes a structural transition at $125 \mathrm{~K}$ from the tetragonal $P 4_{2} / m$ to the triclinic $P \overline{1}$ symmetry [14]. This tetragonal to triclinic transition is not accompanied by any volume discontinuity and only involves a very weak modification of the lattice parameters from $a=$ $8.325(1) \AA$ and $c=5.090(1) \AA$ at $300 \mathrm{~K}$ to $a=8.292(1) \AA$, $b=8.280(1) \AA$, and $c=5.067(1) \AA$ together with unit-cell angles of $\alpha=90.44(1)^{\circ}, \beta=90.60(1)^{\circ}$, and $\gamma=90.07(1)^{\circ}$ at $4 \mathrm{~K}$. In order to evaluate the impact of this distortion on the magnetic couplings, broken-symmetry calculations performed using the experimental structure determined at $300 \mathrm{~K}$ have been extended to a set of 61 structures obtained from the Rietveld refinement of neutron powder diffraction experiments carried out from 2 to $300 \mathrm{~K}$. The results are summarized in Fig. 3(a) where data points are represented by dots and a smooth interpolation using Boltzmann sigmoids [32] is superimposed. It should be mentioned that the weakness of the triclinic distortion close to the transition temperature leads to large standard deviations in the Rietveld refinements and partly explains the noise visible in the results shown in Fig. 3. Moreover, the use of this interpolation was motivated by the fact that, in the $125-140 \mathrm{~K}$ range, neutron refinements were carried out using the tetragonal symmetry even if the synchrotron x-ray diffraction patterns revealed the presence of large microstrain effects (lattice parameter fluctuations). These fluctuations indeed indicate that substantial deviations of local bond lengths and angles from
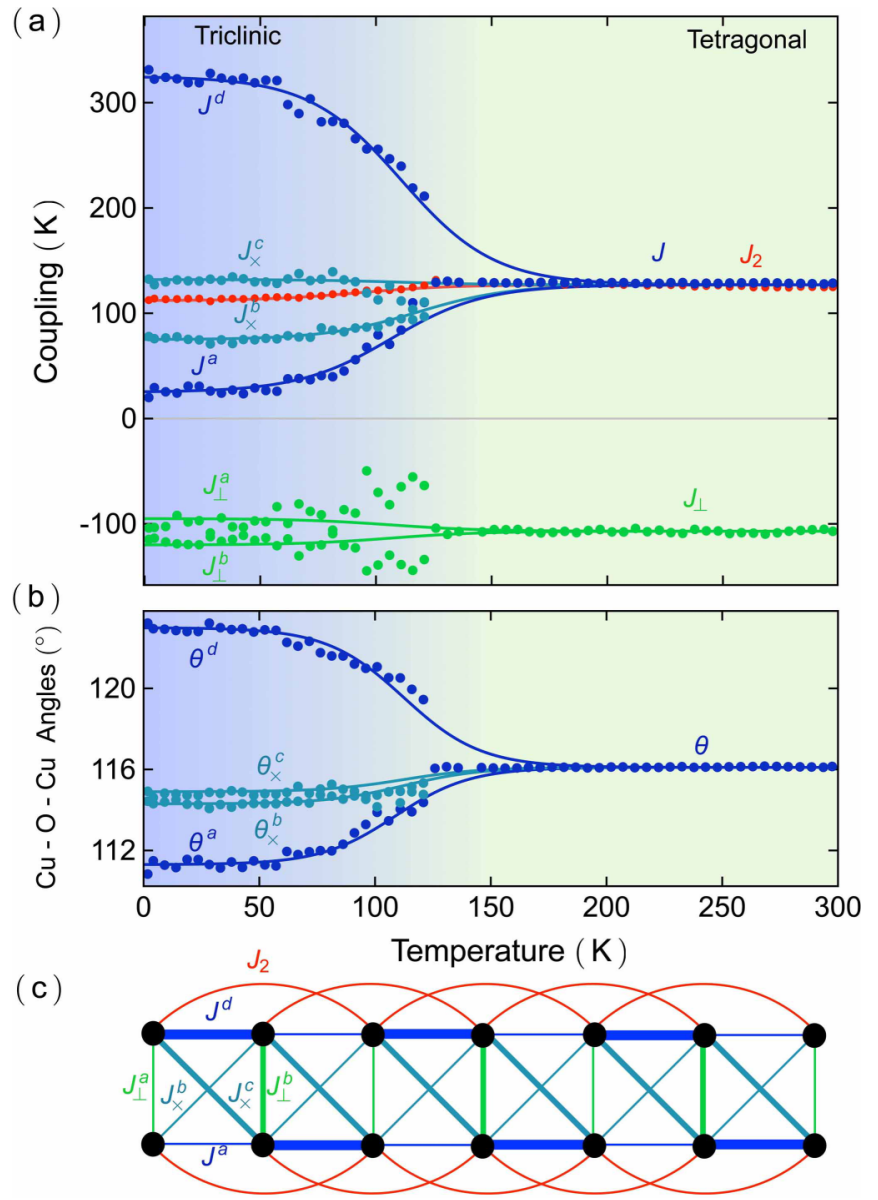

FIG. 3. (a) Temperature dependence of the magnetic couplings in $\mathrm{Li}_{2} \mathrm{Cu}_{2} \mathrm{O}\left(\mathrm{SO}_{4}\right)_{2}$ calculated in $\mathrm{GGA}+U$. Data points are represented with dots; the Boltzmann sigmoid fits are represented with lines. (b) Experimental temperature dependence of the interplatelet $\mathrm{Cu}-\mathrm{O}-\mathrm{Cu}$ superexchange angles. (c) Schematic representation of the staggered dimer structure deduced for the triclinic phase (below the transition temperature of $\sim 125 \mathrm{~K}$ ). The inequivalent triclinic interactions along the legs $J^{a}$ and $J^{d}$, diagonal interactions between the legs $J_{\times}^{b}$ and $J_{\times}^{c}$, and between the legs along the rungs $J_{\perp}^{a}$ and $J_{\perp}^{b}$, are represented in dark blue, blue, and green, respectively. The same color convention has been used for the superexchange angles. The NNN interaction along the legs $J_{2}$ is represented in red.

the ideal tetragonal structure already occur above the transition temperature [14].

Whereas the triclinic distortion has only a marginal effect on $J_{\perp}$ and $J_{2}$ [33], it drastically impacts the interplatelet coupling $J$. Firstly, the crystal symmetry lowering in the triclinic phase lifts its original degeneracy, leading to four distinct couplings instead of a single one in the tetragonal phase. Secondly, each of these couplings follows a distinct trend as the temperature is lowered: two of them largely reduce their amplitude $\left(J^{a}\right.$ and $\left.J_{\times}^{b}\right)$, one remains almost constant $\left(J_{\times}^{c}\right)$, whereas the last one experiences a drastic increase $\left(J^{d}\right)$, raising its amplitude to almost three times its room-temperature value. This very strong variation of the predicted magnetic couplings with the temperature is not surprising if we consider the detailed evolution of the atomic arrangement inside the unit cell. Indeed, although the triclinic distortion has only a modest 


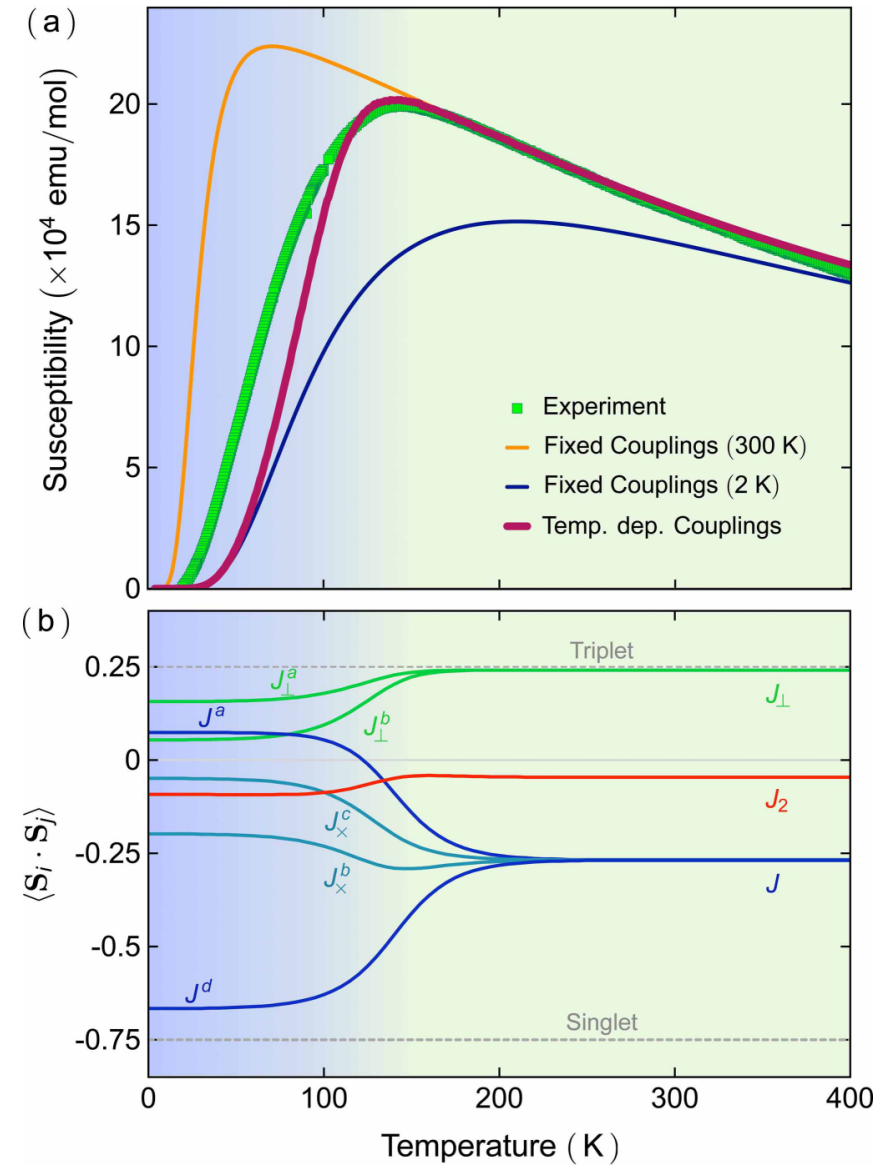

FIG. 4. (a) Temperature dependence of the magnetic susceptibility of $\mathrm{Li}_{2} \mathrm{Cu}_{2} \mathrm{O}\left(\mathrm{SO}_{4}\right)_{2}$. The experimental points are shown in green. The calculations performed with the fixed couplings corresponding to the high-temperature $(300 \mathrm{~K})$ structure are shown in orange, with the fixed couplings corresponding to the low-temperature $(2 \mathrm{~K})$ structure in dark blue, and with temperature-dependent couplings shown by a thick purple line. (b) Temperature dependence of the spin correlations $\left\langle\mathbf{S}_{i} \cdot \mathbf{S}_{j}\right\rangle$ calculated using Lanczos diagonalization. The labels are the associated exchange interactions and the colors follow the convention used in Fig. 3.

impact on the lattice parameters, it involves a sizable variation of the interplatelet $\mathrm{Cu}-\mathrm{O}-\mathrm{Cu}$ superexchange angle, as shown in Fig. 3(b). As the $\mathrm{Cu}-3 d / \mathrm{O}-2 p$ hopping is directly related to this angle and as the superexchange interaction directly scales with this integral, the amplitude of the resulting AFM couplings correlates exactly with the $\mathrm{Cu}-\mathrm{O}-\mathrm{Cu}$ angle [see Figs. 3(a) and 3 (b)]. The picture resulting from these calculations is therefore that the weak triclinic structural distortion involves a strong magnetic dimerization. The resulting schematic staggered $S=$ $1 / 2$ dimer magnetic structure, lifting most of the frustration, is shown in Fig. 3(c). The temperature dependence of the expectation values of the spin correlations, based on the smooth interpolations of the magnetic couplings presented in
Fig. 3(a), is shown in Fig. 4(b). The magnetic dimerization is evidenced at low temperature where the spin correlation associated with $J^{d}$ reaches a value of -0.666 , very close to the value of an isolated singlet, -0.750 . The weaker magnetic correlations between the dimers result from the remaining interactions.

In order to verify the validity of these findings, the temperature dependence of the magnetic susceptibility has been calculated using full diagonalization on a finite lattice of 16 spins and imposing periodic boundary conditions (finitesize effects are relatively small; see Supplemental Material [29]). Three models based on the couplings obtained by DFT have been confronted to the experiments [14], corresponding respectively to (i) a set of fixed, i.e., temperature-independent couplings determined from the experimental tetragonal structure determined at $300 \mathrm{~K}$; (ii) a set of fixed couplings determined from the experimental triclinic structure at $2 \mathrm{~K}$; and (iii) a set of variable, i.e., temperature-dependent, couplings based on the interpolations presented in Fig. 3(a). The results are shown in Fig. 4(a) where the fit of the experimental data is solely based on the adjustment of the $g$ factor, set to $g \simeq 2.10$, a reasonable value for $\mathrm{Cu}^{2+}$ [34]. The best agreement is clearly obtained for the model with the temperature-dependent exchange interactions, confirming the large impact of the structural distortion on the magnetism of this compound. Remaining discrepancies, particularly visible at low temperature through a substantial overestimation of the spin gap, are directly attributable to the semiquantitative nature of the magnetic couplings calculated in DFT. These quantities indeed strongly depend on the approximations used in the treatment of exchange and correlation and are often overestimated [35].

In summary, $\mathrm{Li}_{2} \mathrm{Cu}_{2} \mathrm{O}\left(\mathrm{SO}_{4}\right)_{2}$ appears, in its tetragonal structure, as a very rare realization of a $S=1 / 2$ frustrated two-leg spin ladder where frustration arises from competing NN and NNN interactions along the legs. The unusual triclinic distortion occurring in this compound at about $125 \mathrm{~K}$ is accompanied by a drastic modification of its magnetic properties. We indeed showed that a strong magnetoelastic coupling is responsible for the formation of a staggered $S=$ $1 / 2$ dimer structure, lifting most of the magnetic frustration. This work should motivate further experimental investigations of $\mathrm{Li}_{2} \mathrm{Cu}_{2} \mathrm{O}\left(\mathrm{SO}_{4}\right)_{2}$, as this compound appears as a prototypical material system to study the physics of spin-lattice coupling in quasi-1D frustrated quantum antiferromagnets.

This work was granted access to the HPC resources of IDRIS under the allocation 2016-i2016097218 made by GENCI (Grand Equipement National de Calcul Intensif). This work was supported by French state funds managed by the ANR within the Investissements d'Avenir programme under reference ANR-11-IDEX-0004-02, and more specifically within the framework of the Cluster of Excellence MATISSE led by Sorbonne Universités.
[1] Introduction to Frustrated Magnetism, edited by C. Lacroix, P. Mendels, and F. Mila, Springer Series in Solid-State Sciences Vol. 164 (Springer, New York, 2011).
[2] M. P. Gelfand, Phys. Rev. B 43, 8644 (1991).

[3] Y. Xian, Phys. Rev. B 52, 12485 (1995). 
[4] Zheng Weihong, V. Kotov, and J. Oitmaa, Phys. Rev. B 57, 11439 (1998).

[5] O. A. Starykh and L. Balents, Phys. Rev. Lett. 93, 127202 (2004).

[6] B. W. Ramakko and M. Azzouz, Phys. Rev. B 76, 064419 (2007).

[7] T. Vekua and A. Honecker, Phys. Rev. B 73, 214427 (2006).

[8] M. Azuma, Z. Hiroi, M. Takano, K. Ishida, and Y. Kitaoka, Phys. Rev. Lett. 73, 3463 (1994).

[9] M. Matsuda and K. Katsumata, Phys. Rev. B 53, 12201 (1996).

[10] R. S. Eccleston, M. Uehara, J. Akimitsu, H. Eisaki, N. Motoyama, and S.-I. Uchida, Phys. Rev. Lett. 81, 1702 (1998).

[11] B. Koteswararao, S. Salunke, A. V. Mahajan, I. Dasgupta, and J. Bobroff, Phys. Rev. B 76, 052402 (2007).

[12] A. A. Tsirlin, I. Rousochatzakis, D. Kasinathan, O. Janson, R. Nath, F. Weickert, C. Geibel, A. M. Läuchli, and H. Rosner, Phys. Rev. B 82, 144426 (2010).

[13] M. Sun, G. Rousse, A. M. Abakumov, M. Saubanère, M.-L. Doublet, J. Rodríguez-Carvajal, G. Van Tendeloo, and J.-M. Tarascon, Chem. Mater. 27, 3077 (2015).

[14] G. Rousse, J. Rodríguez-Carvajal, C. Giacobbe, M. Sun, O. Vaccarelli, and G. Radtke, Phys. Rev. B 95, 144103 (2017).

[15] A. Saúl and G. Radtke, Phys. Rev. Lett. 106, 177203 (2011).

[16] P. Gianozzi et al., J. Phys.: Condens. Matter 21, 395502 (2009).

[17] J. P. Perdew, K. Burke, and M. Ernzerhof, Phys. Rev. Lett. 77, 3865 (1996).

[18] K. F. Garrity, J. W. Bennett, K. M. Rabe, and D. Vanderbilt, Comput. Mater. Sci. 81, 446 (2014).

[19] N. Marzari and D. Vanderbilt, Phys. Rev. B 56, 12847 (1997).

[20] A. A. Mostofi, J. R. Yatesb, Y.-S. Lee, I. Souza, D. Vanderbilt, and N. Marzari, Comput. Phys. Commun. 178, 685 (2008).

[21] J. B. Goodenough, Magnetism and the Chemical Bond (Interscience, New York, 1963).

[22] W. Geertsma, Physica B 164, 241 (1990).
[23] H. Eskes and J. H. Jefferson, Phys. Rev. B 48, 9788 (1993).

[24] D. A. Tennant, S. E. Nagler, A. W. Garrett, T. Barnes, and C. C. Torardi, Phys. Rev. Lett. 78, 4998 (1997).

[25] Van H. Crowford, H. W. Richardson, J. R. Wasson, D. J. Hodgson, and W. E. Hatfield, Inorg. Chem. 15, 2107 (1976).

[26] A. Saúl and G. Radtke, Phys. Rev. B 89, 104414 (2014).

[27] M. Cococcioni and S. de Gironcoli, Phys. Rev. B 71, 035105 (2005).

[28] H. J. Kulik, M. Cococcioni, D. A. Scherlis, and N. Marzari, Phys. Rev. Lett. 97, 103001 (2006).

[29] See Supplemental Material at http://link.aps.org/supplemental/ 10.1103/PhysRevB.96.180406, which includes Refs. $[15,30,31]$, for a detailed description of the broken symmetry formalism employed to evaluate the magnetic couplings and the finite size effects on the magnetic susceptibility.

[30] G. Radtke, A. Saúl, H. A. Dabkowska, M. B. Salamon, and M. Jaime, Proc. Natl. Acad. Sci. USA 112, 1971 (2015).

[31] M. Okada, H. Tanaka, N. Kurita, K. Johmoto, H. Uekusa, A. Miyake, M. Tokunaga, S. Nishimoto, M. Nakamura, M. Jaime, G. Radtke, and A. Saúl, Phys. Rev. B 94, 094421 (2016).

[32] L. D. Casto, A. J. Clune, M. O. Yokosuk, J. L. Musfeldt, T. J. Williams, H. L. Zhuang, M.-W. Lin, K. Xiao, R. G. Hennig, B. C. Sales, J.-Q. Yan, and D. Mandrus, APL Mater. 3, 041515 (2015).

[33] Note that, strictly, two distinct $J_{2}$ couplings, between crystallographically inequivalent $\mathrm{Cu}(1)$ and $\mathrm{Cu}(2)$ ions [14], respectively, should be considered in the triclinic phase. They are, however, numerically so close that they have been considered as a unique coupling here.

[34] F. E. Mabbs and D. J. Machin, Magnetism and Transition Metal Complexes (Dover, New York, 2008).

[35] R. L. Martin and F. Illas, Phys. Rev. Lett. 79, 1539 (1997). 